\title{
Marion Eckertz-Höfer, Präsidentin des Bundesverwaltungsgerichts
}

\author{
Juristinnen machen Karriere - wir stellen sie vor
}

Das Interview wurde geführt von Marianne Grabrucker, Vors. Richterin am BPatG a.D., München, im August 2013.

Frau Eckertz-Höfer, Sie gehören zu der Generation, die um das Jahr '68 Abitur gemacht hat und ihr Studium begann. Was hat Sie bewogen, Jura zu studieren?

1968 lebte ich noch voll im Bewusstsein der Kriegszeit und der harten Existenz meiner Eltern in den Nachkriegsjahren. Sie hatten - und konnten wohl gar nicht anders - diese Erfahrungen als Gefühl an meinen Bruder und mich weitergegeben. Die Gespräche über die Verbrechen der Nazizeit und die jüdischen Freunde meines Vaters waren für mich absolut prägend. Ich war deshalb als Jugendliche von dem Gedanken durchdrungen: Nie wieder Krieg, nie wieder Verbrechen in deutschem Namen! Diese Motive haben sich im Laufe meines Lebens auch nicht geändert. Frauenpolitik kam später hinzu, ohne das andere zu verdrängen. Beruflich wollte ich in einem Umfeld arbeiten, in dem ich Entscheidungen zumindest mit beeinflussen kann, möglicherweise in der Politik. Jura schien mir damals - das sehe ich heute nicht anders - ein sinnvolles Rüstzeug dafür zu sein.

Sie erinnern sich an das damals häufig zu hörende Schlagwort vom „Marsch durch die Institutionen“ - mit dem Ziel einer veränderten Gesellschaft. Sie sind nun durch viele Ämter hindurch „marschiert“ und haben es an die Spitze geschafft. Was halten Sie von dieser Vorstellung 45 Jahre später?

Der propagierte „Marsch durch die Institutionen“ dürfte vor allem scherzhaft gemeint gewesen sein. So habe ich das zumindest verstanden. Unterwanderung als Lebensplan? Das kann kaum funktionieren. Wichtiger war mir der Gedanke, in Umbruchphasen Verantwortung zu tragen und sie zu nutzen - wenn notwendig. Ich habe es nie als selbstverständlich angesehen, dass wir in einer Demokratie und in Frieden leben und dass dies auch so bleibt. Meine Eltern scheinen hier wohl auf mich abgefärbt zu haben! Anders als sie wollte ich aber nicht die Fäuste nur in der Tasche ballen, wenn es wirklich zu Bedrohungen von Demokratie und Rechtsstaat käme. Deshalb war ich immer sehr aufmerksam, wenn es um rechtsradikale Strömungen ging. Einen Mördertrupp wie den NSU, zu dem der Strafprozess gerade in München stattfindet, hätte ich mir allerdings kaum vorstellen können. Die Wirklichkeit ist ja nicht selten schrecklicher als die Phantasie! Inzwischen sehe ich mit Freude, dass Justiz und Rechtsstaat in Deutschland gefestigt sind. Dies wird auch weltweit anerkannt. Bei meinen häufigen Auslandsbesuchen vernehme ich viel Anerkennung und Respekt für Deutschland und seine Justiz. Unsere Gerichtsentscheidungen stoßen auf ein erstaunliches Interesse, trotz der Sprachbarrieren und zwar nicht nur, aber doch auch in Ländern, die auf der Suche nach demokratischer Neuordnung sind.
Marion Eckertz-Höfer, Präsidentin des Bundesverwaltungsgerichts, geboren 23. November 1948 in Oldenburg (i.O.)

1967 Abitur (Duisburg)

1974 Erstes Juristisches Staatsexamen in Tübingen (nach einem Studium der Rechtswissenschaften und der Volkswirtschaft - Saarbrücken und Tübingen)

1977 Zweites Juristisches Staatsexamen in Stuttgart

1977 Strafrichterin in Heidelberg

1978 Staatsanwältin in Mannheim

1979 Richterin für erstinstanzliche Zivilstreitigkeiten, Landgericht Mannheim

1983 Wissenschaftliche Mitarbeiterin am Bundesverfassungsgericht, Karlsruhe (Dezernat des Bundesverfassungsrichters Dr. Helmut Simon)

1988 Amtschefin (Stellvertreterin der Ministerin/Ministerialdirigentin) des Frauenministeriums in Schleswig-Holstein, Kiel

Von 1991 bis 1993: Persönliche Beauftragte des Innenministers von Schleswig-Holstein in der Verfassungskommission des Bundesrates

1993 Wahl zur Richterin am Bundesverwaltungsgericht (ab 1995 - 2002: auch Gleichstellungsbeauftragte des Gerichts)

2001 Vorsitzende Richterin am BVerwG (Senat für Asyl- und Ausländerrecht, Staatsangehörigkeitsrecht)

2002 Vizepräsidentin des BVerwG

2007 Präsidentin des BVerwG (und Vors. Richterin im Senat für Ausländerrecht)

Fühlen Sie sich im Vergleich zu anderen Frauen Ihres Alters mit ähnlichem Ausbildungsabschluss eher als Solitär oder denken Sie, dass Ihr Lebensweg typisch ist?

Im Vergleich zu den Klassenkameradinnen in meiner Mädchenschule bin ich natürlich Solitär. Für die meisten aus meiner Schule stand die Familiengründung im Vordergrund, ähnlich wie in der Generation meiner Mutter. Meine Mutter pflegte mir allerdings gefühlte tausendmal im Jahr zu sagen: Das Wichtigste im Leben ist ein guter Beruf, alles andere ergibt sich. Auch wenn ich dies damals als überaus nervig empfand: Es scheint sich in mir festgesetzt zu haben! Insgesamt erging es meiner Frauengeneration viel besser. Ich hatte immerhin schon ein paar weibliche Vorbilder. An der Uni hatte ich zwar keine Professorin erleben dürfen, aber Juristinnen wie Elisabeth Selbert, die Mutter des Art. 3 Abs. 2 GG, oder die Bundesverfassungsrichterinnen Wiltrud Rupp-von Brünneck und Helga Seibert hatten schon Vorbildcharakter für mich. Helga Seibert, die leider viel zu früh verstorben ist, durfte ich selbst noch kennen lernen. Von 
den vielen tollen lebenden Frauen, die mir Vorbild waren und sind, ganz zu schweigen!

\section{Wie kamen Sie denn zur Frauenpolitik?}

Die Beschäftigung mit Frauenpolitik war mehr oder weniger eine „Kopfgeburt“ während meiner Zeit als wissenschaftliche Mitarbeiterin beim Bundesverfassungsgericht. Die erste intensivere Berührung mit dem Thema „Frauendiskriminierung“ kam, als ich mich auf den Besuch einer EU-Frauenkonferenz in der Nähe Brüssels vorbereitete. Ein Kollege hatte mich vorgeschlagen - mit der Begründung, ich hätte doch immer so flotte Sprüche auf der Lippe. Da begann ich, mich einzulesen und ein AHA-Erlebnis nach dem anderen stellte sich ein! Mir erschloss sich eine völlig neue Welt, die ich vorher so nicht wahrgenommen hatte oder nicht hatte wahrnehmen wollen, die mir aber vertraut war. Ich wusste sofort, dies ist ein Thema, um das ich mich längerfristig kümmern muss. Beim BVerfG gab es dazu dann auch die Gelegenheiten: „Mein“ Verfassungsrichter Helmut Simon hatte mich z.B. - obwohl er selbst nicht Berichterstatter war - in die dezernatsinterne Diskussion zur Rentenaltersentscheidung einbezogen. Das war der Fall des alleinerziehenden Wittwers, der meinte - wie damals nur für Frauen gesetzlich zugelassen, schon mit 60 Jahren sein Altersruhegeld beanspruchen zu dürfen. Frage war: Darf der Gesetzgeber berücksichtigen - und diesen Nachteil über eine unterschiedliche Regelung des Rentenalters ausgleichen -, dass die Doppelbelastung durch Kindererziehung typischerweise deutlich mehr Frauen als Männer betrifft. Dies erlaubten ihm damals Art. 3 Abs. 2 GG und das Sozialstaatsprinzip. Heute ist dies europarechtlich untersagt. Diesen Fall positiver Diskriminierung verarbeitete ich dann in der Festschrift für Helmut Simon. So kam Eines zum Anderen - und das Thema ließ mich nicht mehr los.

Nach der Zeit am Bundesverfassungsgericht und einem kurzen Intermezzo beim Landgericht in Mannheim, an dem ich vorher schon tätig gewesen war, wurde ich - wohl wegen meiner Publikationen auf diesem Gebiet - gefragt, ob ich denn nicht in Kiel in der Regierung Engholm 1988 Amtschefin/Ministerialdirigentin im dort neu gegründeten Frauenministerium werden wollte. Es sollte das Erste in der BRD sein und mir war sofort klar, dass ich wollte!

\section{Was waren Ihre prägendsten Eindrücke aus dieser Zeit?}

Es galt, ein kleines durchsetzungsstarkes Frauenministerium aus dem Nichts aufzubauen. Ich hatte das Glück, mit Gisela Böhrk, einer politisch erfahrenen, gut vernetzten und ideenreichen Frauenministerin zusammen zu arbeiten. Sie ging das Frauenthema als Querschnittsthema und zwar von der arbeitsund wirtschaftspolitischen Seite her an und initiierte z.B. ein Wiedereinstiegsprogramm für Frauen, das aus einer Kombination von ortsnaher Beratung im ländlichen Raum, Information und Vernetzung mit örtlichen Arbeitgebern bestand. Für ein sehr vergleichbares Projekt auf Bundesebene hat - kaum sind 25 Jahre vorbei - in diesem Jahr die derzeit (auch) für Frauen zuständige Bundesministerin einen Preis der Vereinten Nationen erhalten. Darüber hinaus waren wir das erste Land, in dem flächendeckend Gleichstellungsbeauftragte in sämtlichen Behörden, Kommunen und Kreisen eingerichtet wurden. Der erste von uns organisierte Kongress aller Gleichstellungsbeauftragten war beeindruckend: Ungefähr 1000 Frauen trafen sich, die alle amtlich damit beschäftigt waren, bei Ausbildung, Einstellung und täglicher Arbeit auf Gleichbehandlung von Frauen - am Maßstab der Behandlung von Männern - zu achten. Dies war auch vielfach nötig! Übrigens sahen sich die wenigsten als Feministinnen. Sie wollten aber ihre Aufgabe gut machen und wie von selbst entstand dann vielfach das Interesse, die Sensibilität und die erforderliche Professionalität. Im Ministerium war ich vor allem dafür verantwortlich, dass die ersonnenen Rechtsvorschriften zur Gleichstellung vor den Gerichten Stand hielten, was gelang. Als Mitglied der Staatssekretärskonferenz (ohne wegen der Kleinheit des Ministeriums formell Staatssekretärin zu sein) war ich in politische Leitentscheidungen auch außerhalb von Frauenpolitik eingebunden. Mein Rat in verfassungsrechtlichen Einzelfragen wurde auch von anderen Ressorts erbeten. Eine 100-Stunden-Woche war keine Ausnahme, eher die Regel.

Mit dem Mauerfall am 9. November 1989 trat dann noch eine „fünfte“ Dimension hinzu. „Das Wort veraltete einem im Munde“, so überschlugen sich damals die Ereignisse. Die Länder wurden von der Bundesregierung zu den im Einigungsvertrag zu regelnden Materien angehört. In den Monaten vor Abschluss des Einigungsvertrags Ende August 1990 erreichten mich - und die anderen Ressorts - fast sämtliche Unterlagen der Vertragsverhandlungen. Ich lebte Zeitgeschichte pur, wenn auch mit viel Papier verbunden! Mein Part war es, die frauenrelevanten Teile zu überprüfen und zu bewerten. Ich habe selten eine spannendere Zeit erlebt! Dann trat noch hinzu, dass der Bundesrat mit Beschluss vom 1. März 1991 die Kommission „Verfassungsreform“ ins Leben gerufen hatte. Sie sollte auf Länderebene die Gemeinsame Verfassungskommission von Bundestag und Bundesrat inhaltlich vorbereiten. Als durch das Landeskabinett bestellte persönliche Vertreterin des Innenministers des Landes Schleswig-Holstein vertrat ich das Land im Arbeitsausschuss 2 dieser Kommission und wurde Berichterstatterin der A-Länder für eine Ergänzung des Art. 3 Abs. 2 GG. Der Ergänzungsvorschlag Schleswig-Holsteins lautete „Der Staat gewährleistet die Gleichstellung der Frauen in allen gesellschaftlichen Bereichen " bzw. - nach weiteren Beratungen der A-Länder: „Der Staat hat die Bedingungen für die Gleichstellung von Frauen und Männern in der Gesellschaft zu schaffen. Zum Ausgleich bestehender Ungleichheiten sind Maßnahmen zur Förderung von Frauen zulässig“. Für keinen dieser und weiterer Vorschläge fand sich eine Mehrheit im Bundesrat. Schließlich beschloss die Gemeinsame Verfassungskommission von Bundesrat und Bundestag 1994 die jetzt geltende Ergänzung von Art. 3 Abs. 2 GG: „Der Staat fördert die tatsächliche Durchsetzung der Gleichberechtigung von Frauen und Männern und wirkt auf die Beseitigung bestehender Nachteile hin “. Aber die Debatten insgesamt, insbesondere auch zu den Nicht-Frauenthemen, gehören doch zu den interessanteren Momenten meines Berufslebens. 


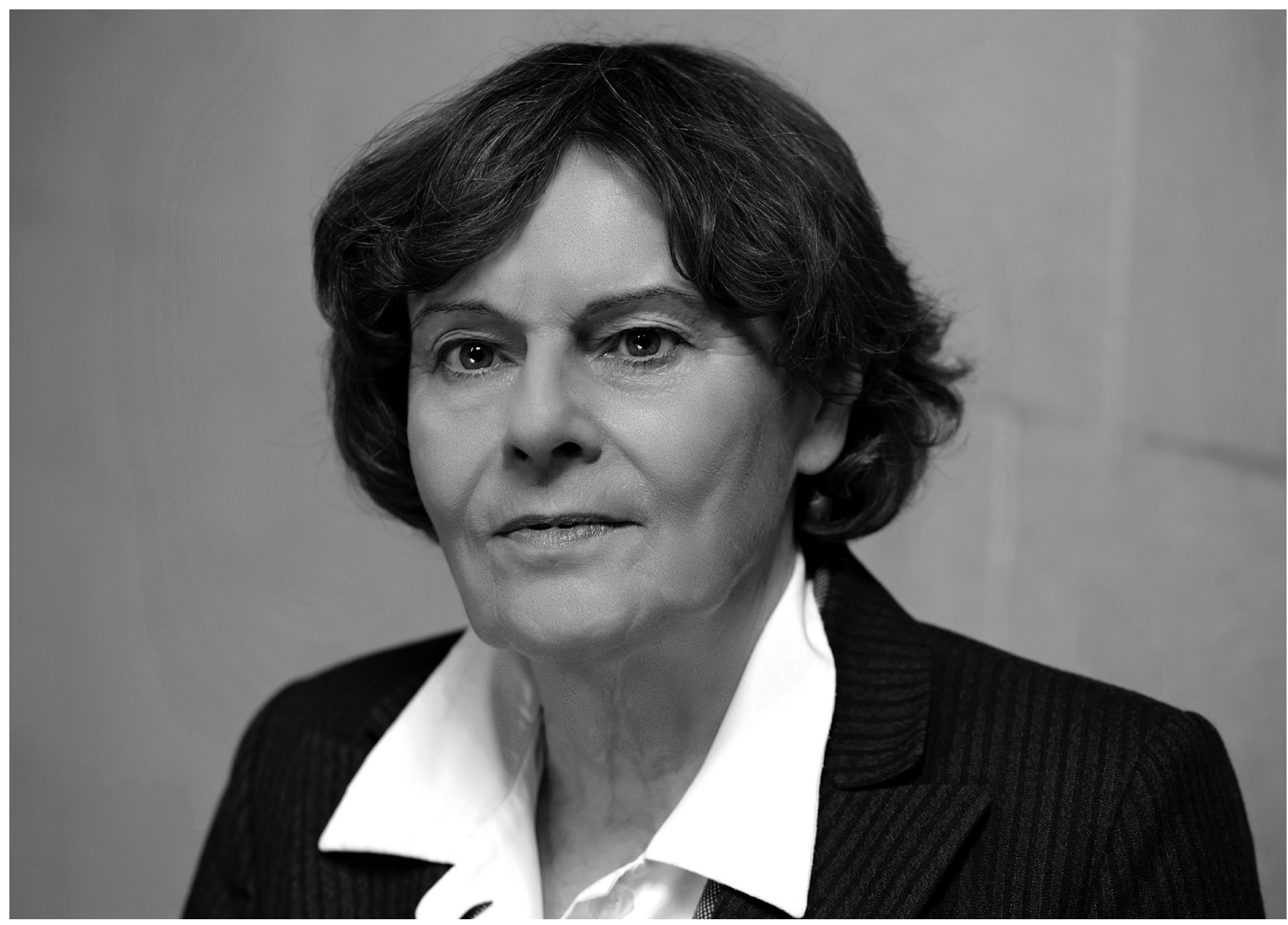

A Marion Eckertz-Höfer, Präsidentin des Bundesverwaltungsgerichts, Foto: Bundesverwaltungsgericht.

\section{Wie war denn der weitere Karriereverlauf?}

Ich hatte von vorneherein meine Zeit in der Frauenpolitik auf ca. fünf Jahre begrenzt. 1993 wurde ich als Richterin für das Bundesverwaltungsgericht vorgeschlagen und zu meiner Überraschung - immerhin war ich nie vorher Verwaltungsrichterin gewesen - auch gewählt. Mein Start war in einem Senat mit hochinteressanten Materien wie z.B. Rundfunk-, Presse- und Telekommunikationsrecht. Der erste Fall als Mitberichterstatterin betraf das Namensrecht, also eine durchaus frauenrelevante Materie, wenn es um den Familiennamen der Kinder aus geschiedenen Ehen geht. Als ab 1994 das Frauenfördergesetz des Bundes mit der Verpflichtung galt, Frauenbeauftragte auch in den Gerichten wählen zu lassen, war das für mich eine schöne Gelegenheit, mein altes Engagement fortzusetzen. Dankenswerterweise wurde ich gewählt und blieb Frauenbeauftragte des Gerichts bis 2002, als ich Vizepräsidentin wurde. Im Juni 2007 wurde ich schließlich zur Präsidentin des Bundesverwaltungsgerichts ernannt.

\section{Im Rückblick gesehen: War die Arbeit der Frauenbewegung er-} folgreich in dem Sinne, dass wir ohne die Feministinnen der 7oer und 8oer Jahre heute nicht diese für Frauen im Vergleich zu den 6oer Jahren deutlich bessere Statistik hätten oder glauben Sie, dass diese Entwicklung auch von alleine stattgefunden hätte? Eines halte ich für sicher: Eine Frauenbewegung ohne Regierungsmacht und ohne Durchsetzungsmechanismen bringt eher wenig. Das hat bereits die erste Frauenbewegung Ende des 19. Jahrhunderts gezeigt. Bloße Regierungsmacht ist allerdings auch nicht zielführend, wie man an einigen der seit den $80 \mathrm{er}$ Jahren auch für Frauen zuständigen Ministerinnen im Bund und in den Ländern sieht. Nur wenige wussten mit dieser Zuständigkeit wirklich etwas anzufangen. Eine inzwischen verbesserte - allerdings noch weit vom anzustrebenden „Normalzustand“ einer hälftigen Beteiligung von Frauen auf allen Ebenen entfernte - Statistik gibt es vor allem im öffentlichen Dienst. Das ist anzuerkennen und wichtig: Denn der öffentliche Dienst hat eine Vorbildfunktion. Für problematisch - damals wie heute - halte ich die Situation in der Privatwirtschaft. $\mathrm{Zu}$ Berufsbeginn ist dies inzwischen wohl nicht mehr so ausgeprägt der Fall: Die Mädchen haben sich durch bessere Schulnoten und gute Examensergebnisse einen Vorsprung verschafft, der aber immer noch selten in Führungspositionen führt. Denn er geht leider schnell mit dem ersten Kind verloren. Die jetzigen Aktionen, um die sich gerade auch der Deutsche Juristinnenbund verdient gemacht hat, mit „Frauen in die Aufsichtsräte“ stehen für Mängel in der gesamten Privatwirtschaft. Ein großer Fehler der Regierung Schröder war es gewesen, Frauenpolitik als „Gedöns“ abzutun. Hätte man vor zehn Jahren den „Entwurf eines Gesetzes zur Gleichstellung der Geschlechter in der Privatwirtschaft" - im Wesentlichen aus der Feder von Heide Pfarr - umgesetzt, so sähe die Privatwirtschaft heute anders 
aus: Nicht weniger effizient, aber ein Stück gerechter! Leider finden auf dieser Welt von alleine wohl nur Naturkatastrophen statt - und auch von diesen sind nicht wenige auf menschliches Verhalten zurückzuführen!

Zu Beginn der neuen Frauenbewegung sprach man davon, dass die „richtigen“ Frauen an die „richtigen Positionen“ kommen müssten, um sodann dafür zu sorgen, dass sich die Verhältnisse gesamtgesellschaftlich zugunsten der Frauen ändern würden. Wie beurteilen Sie die Tatsache, dass nun viele der Frauen, die wichtige Positionen inne haben, eigentlich nie mit der Frauenbewegung oder dem Feminismus zu tun hatten, sich sogar davon distanzieren? Kommt es für Sie dennoch auf das „richtige Bewusstsein“ an oder genügt allein die Statistik?

Es geht um die ungehinderte Teilhabe von Frauen in allen gesellschaftlichen Bereichen und nicht um Meinungskontrolle. Ich habe immer wieder erlebt, dass selbst sich betont „antifeministisch" gebende Frauen durch konkrete Erlebnisse auf einmal wie elektrisiert sind. Entscheidend ist für mich, dass die geeigneten Frauen in die richtigen Positionen kommen und sich auf ihrem Weg nicht größere Hindernisse befinden als bei vergleichbaren Männern.

Was halten Sie von der stets zitierten „Vereinbarkeit von Beruf und Familie für Frauen"? Kommt das nicht eigentlich der Zementierung der hergekommenen Familienstrukturen gleich?

Die Vereinbarkeit von Familie und Beruf ist das Ziel für Männer und Frauen - der öffentliche Dienst zeigt, dass dies geht. Faktisch haben wir natürlich das Problem, dass es überwiegend die Frauen sind, die die - insbesondere arbeitszeitlichen - Vergünstigungen zur Kinderbetreuung in Anspruch nehmen. In der Tat verfestigt dies den Status quo. Inzwischen wissen jedoch auch immer mehr Männer, was sie bei ihren Kindern verpassen, und nehmen Elternzeit oder arbeiten in Teilzeit. Leider sind es noch viel zu wenige. Die Frauen sollten jedoch auf keinen Fall den Beruf um die Familie herum „stricken“ und nur mit halber Seele und halbem Herzen bei der Arbeit sein. Auf diese Weise können sich beruflich kaum die Erfolge einstellen, die sie weiter bringen. Das habe ich auch bei Männern erlebt, die überwiegend berufsfremde Interessen pflegen, was aber viel seltener ist. Sie sind wohl in ihrem Lebensplan immer noch mehr zur Berufstätigkeit hin erzogen und wissen meist genau, dass sie es zu etwas bringen wollen. Wohingegen bei Frauen immer noch die Vorstellung des zweiten „Lebens“ besteht, nämlich dass der Beruf „neben“ der Familie herläuft.

In letzter Zeit ist in der Frauenliteratur häufig zu lesen, dass Frauen zu wenig kämpfen, um im Beruf aufzusteigen. Stimmt das mit lhrer Erfahrung überein?

In Bezug auf beide Geschlechter habe ich immer wieder gesehen, dass es nicht zwingend die Leistungsstärksten sind, die gezielt und auch „ellenbogenmäßig“ an ihrer Karriere basteln. Ist ein Unternehmen oder eine Behörde gut geführt, sind tatsächliche stetig gute Leistungen am Ende doch entscheidender. Das bloße
Eigenlob, man sei „die Beste“, besteht den Realitätstest eher selten. Aber Frauen sollten natürlich nicht sagen: „ach, ich weiß nicht, ob ich das kann", sondern einfach probieren und sich einsetzen. Es wird kaum je etwas Unmögliches verlangt!

\section{Was halten Sie denn von der Quote?}

Unser damaliges schleswig-holsteinisches Gleichstellungsgesetz hat auf die leistungsabhängige Quote gesetzt, also: Bei gleicher Leistung haben Frauen den Vorzug, sofern es um eine Stelle geht, in der Männer überrepräsentiert sind. Im Bund gilt heute Vergleichbares. Leider ist eine solche Regelung leicht über entsprechende Beurteilungen zu unterlaufen, auch wenn hier durch die Gleichstellungsbeauftragten und die Rechtsprechung die Kontrollen besser geworden sind. Die sanktionierten Zielquoten in dem von Heide Pfarr verantworteten hessischen Gleichberechtigungsgesetz versuchten in höchst origineller Weise Umgehungsmöglichkeiten zu erschweren. Leider wurde in keinem der Länder eine begleitende Forschung etabliert, so dass wir nicht wissen, ob es messbare Unterschiede im Erfolg dieser beiden Wege gibt. Für die Privatwirtschaft - ab einer bestimmten Unternehmensgröße - wäre wohl der Weg der Zielquoten zu bevorzugen.

Übrigens bin ich zu einer Zeit in den Beruf eingestiegen, als es für Führungspositionen, auch im öffentlichen Dienst, recht klar eine Männerquote gab - am liebsten 100 Prozent. Da waren natürlich auch viele ungeeignete Männer darunter. Diese „Luschenquote“ haben Frauen wohl noch nirgendwo erreicht. Für die heute propagierte Diversität sind mehr Frauen überall erforderlich. Ich bin mir sicher, dass sie in Sachen „Luschenquote“ die jahrzehntelangen Zielwerte von Männern nie werden toppen können und wohl auch nicht wollen. Jedenfalls gäbe es an den obersten Bundesgerichten noch Verbesserungsmöglichkeiten, auch wenn sich die Frauenquote am BVerwG seit meinen Anfängen im Jahr 1993 um mehr als 20 Prozent erhöht hat. Hier ist der Richterwahlausschuss gefordert: Leider wird dort das Bundesgleichstellungsgesetz mit seiner leistungsabhängigen Quote nicht beachtet, von den nicht ganz seltenen Verstößen gegen den Leistungsgrundsatz des Art. 33 Abs. 2 GG, auch bei Männern, einmal abgesehen. Es hätte jedenfalls deutlich mehr gute Frauen auf den Vorschlagslisten gegeben, als über all die Jahre gewählt wurden. Die Betonung muss dabei immer auf „gut“ liegen, für Frauen ebenso wie für Männer.

\section{Gibt es für Sie als Präsidentin des Gerichts überhaupt eine Ein- flussmöglichkeit im Hinblick auf die Förderung von Frauen?} Ich habe wenig Einfluss im richterlichen Bereich. Das bedeutet aber nicht, dass ich untätig bin: Lerne ich eine gute Frau als wissenschaftliche Mitarbeiterin bei uns am Gericht kennen, so nehme ich z.B. Kontakt auf zu dem Präsidenten ihres Herkunftsgerichts, um frühzeitig zu lancieren, dass ich jemand für sehr geeignet für unser Gericht hielte. Eigentlich sind dies typischerweise Frauen, die auch in ihrem Heimatgericht schon als „top“ aufgefallen sind, und ich renne offene Türen ein. Das politische Umfeld, das weniger 
leistungsbezogen denkt, stellt eine härtere Nuss zu knacken dar. Ich tue jedenfalls alles, um die Chancen solcher Frauen zu verbessern. Einige meiner „Anstrengungen“ sind zu erfolgreichen Richterinnen meines Gerichts geworden. Es hätten aber mehr sein können! Im nichtrichterlichen Bereich genügt es typischerweise, wirklich das Leistungsprinzip zu beachten. Da stehen Frauen eigentlich nie zurück. Von Vorteil ist hier unser Sitz in Leipzig: Frauen mit Wurzeln in der DDR oder von entsprechenden Müttern erzogen, haben doch nicht selten ein ganz anderes Verhältnis zum Beruf als Frauen „im Westen“. Von ihnen geht kaum Eine für drei oder mehr Jahre in Kinderpause.

\section{Was würden Sie heute den jüngeren Frauen mit auf den Weg} geben?

Macht eine gute Ausbildung, übt lebenslanges Lernen und ergreift die Chancen, die sich bieten! Vor allem: Nichts von dem, was Frauen heute erreicht haben, hat sich quasi naturwüchsig und von alleine ergeben. Es bedarf weiter der energischen und tüchtigen Frauen. Also: Engagiert Euch! 